\title{
Patellar Surface Strain
}

\author{
Steven A. Goldstein, Edward Coale, Arnold-Peter C. Weiss, Mark Grossnickle, \\ Bruce Meller, and Larry S. Matthews
}

\begin{abstract}
Biomechanics, Trauma, and Sports Medicine Laboratory, Section of Orthopaedic Surgery, Department of Surgery, University of Michigan, Ann Arbor, Michigan, U.S.A.
\end{abstract}

\begin{abstract}
Summary: We measured longitudinal surface strains on the anterior patella at controlled simulated quadriceps loads, quadriceps angles, and knee flexion angles. We studied both the natural patella and patellas resurfaced with domed, bifaceted, and metal backed polyethylene components. Twelve matched pairs of fresh human cadaver knee specimens were tested. Midanterior longitudinal patellar strains were greatest at knee flexion angles at 45 to $60^{\circ}$ of knee flexion. Alterations in quadriceps angle medially and laterally did not decrease recorded strain values significantly. Polyethylene patellar prostheses increased patellar strains but a bifaceted component or "total contact" design minimized this effect. Metal support for the prosthetic patella decreased the recorded surface strains. This study of patellar surface strain at the three midline locations provides experimental results that may prove useful for design and validation of analytic models of the patella. The study also suggests that patellar surface strain measures may provide a method for evaluating the effects of patellar prosthesis design and resurfacing procedures. Key Words: Patellar strain - Total joint arthroplasty.
\end{abstract}

Fractures of normal patellas result not only from major trauma but also with high level muscular exertion $(9,10,17,26)$. This suggests that at least intermittently the patella functions at near failure strain levels. Patellar fracture after total knee arthroplasty has also been reported as a complication for many major clinical series in which a patella resurfacing prosthesis has been implanted $(1,27)$. Implantation of patellar components frequently requires removal of substantial bone from the patella to provide secure fixation. These techniques may significantly reduce the overall strength of the patella and increase the risk of fracture $(7,20)$. Most of the operative procedures used for the treatment of chondromalacia or patellar malalignment syndromes $(3,4,15,18)$ alter the direction of the major tensile

Address correspondence and reprint requests to Dr. S. A. Goldstein at Section of Orthopaedic Surgery, 2912 B Taubman, Box 0328, University Hospital, Ann Arbor, MI 48109, U.S.A. forces acting on the patella. These changes must alter not only the direction but likely also the magnitudes of local strain experienced by the patella.

Past studies on patellar mechanics, both experimental and analytical, have largely relied on the fundamental works of Morrison (21) and Smidt (25) regarding the mechanics and kinematics of the tibiofemoral articulation to derive further knowledge of the patellofemoral joint. Reports of patellofemoral contact areas $(11,19)$, patellofemoral forces $(3,6,12-14,19,24)$, and the resulting contact stresses $(3,11,19,20,28)$ are also available.

More recently, a patellar model with some experimental substantiation has been developed to study the adaptive behavior of the patellar trabecular bone (8). Although this model may provide predictions of patellar stress contours with function, disease, and surgical alteration, there is still little or no direct experimentally derived information regarding patellar strain.

The purpose of this study was to measure the 
surface strain on the anterior patellar surface as a function of knee flexion angle, quadriceps resultant force angle ( $Q$ angle), and quadriceps force. We also wished to assess the effects of prosthetic resurfacing of the patella by evaluating the effects of prosthetic surface design, fixation peg location, and prosthetic materials on patellar surface strain.

\section{MATERIALS AND METHODS}

Twenty-four fresh human cadaver knee joints from 12 individuals (four women, eight men, ages between 55 and 78 years) were obtained within $24 \mathrm{~h}$ after donation. The quadriceps mechanisms and all capsular and ligamentous structures were preserved. Although mild degenerative changes had occurred in most, no specimen demonstrated severe pathology. All specimens were stored at $-20^{\circ} \mathrm{C}$ in sealed containers until testing. During testing the specimens were thawed to room temperature, wrapped in towels, and kept moist with Ringer's lactate solution. Each knee was refrozen between experimental protocols. Throughout the entire experiment, environmental exposures for all specimens were the same.

Our experimental model involved the careful application of miniature strain gages to proximal, middle, and distal locations on the midvertical anterior surface of the patella. A 3 by $1 \mathrm{~cm}$ area of bone was carefully exposed on each patella through a midpatellar incision with reflection of the soft tissue using a periosteal elevator (Fig. 1). The exposed surface was hand ground with 400 grit silicone carbide paper. Three single 0.030 inch 120

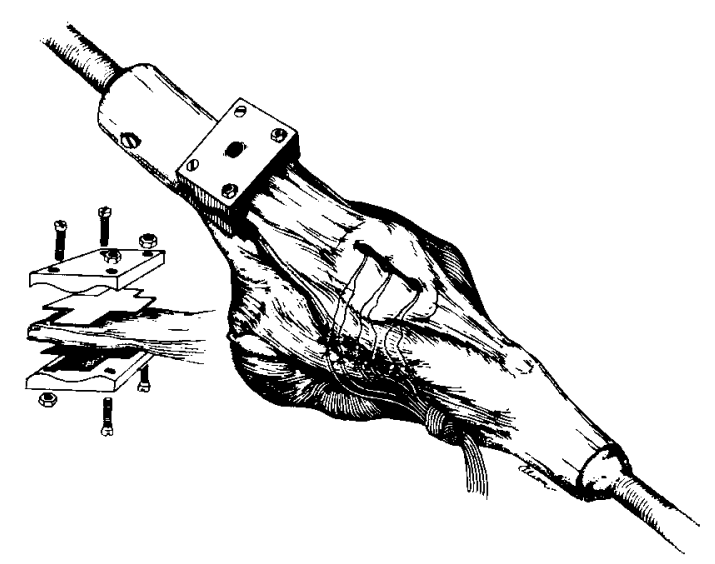

FIG. 1. The three strain gages were fixed to the anterior surface of the patellas. After affixing the gages, the reflected tissue was sutured together. The quadriceps clamp is also illustrated. ohm resistive foil strain gages were cemented to the proximal, middle, and distal poles of the exposed bone using a cyanoacrylic adhesive.

After placement of the gages and attachment of the associated connecting wires, the tissue was sutured back in place. A specially designed tendon clamp (Fig. 1) was attached to the remnants of the quadriceps mechanism and threaded rods were cemented into the femoral and tibial segments for incorporation of the knee into the experimental loading machine (Fig. 2).

In a preliminary study (5) three rosette strain gages were attached to similar locations on seven cadaver knee joints. The results of this study demonstrated that the principal strain directions were essentially longitudinal with the long axis of the patella. Due to the extent and complexity of the experiments and in an effort to reduce the preparation and exposure time of the specimens, the present studies utilized only three longitudinal gages. All tests were conducted at room temperature with the knees kept moist.

A testing machine was developed to grasp the femur securely while allowing the flexion angle of the knee to be predetermined and fixed. The tendon clamp attached to the ram of an Instron Model 1000

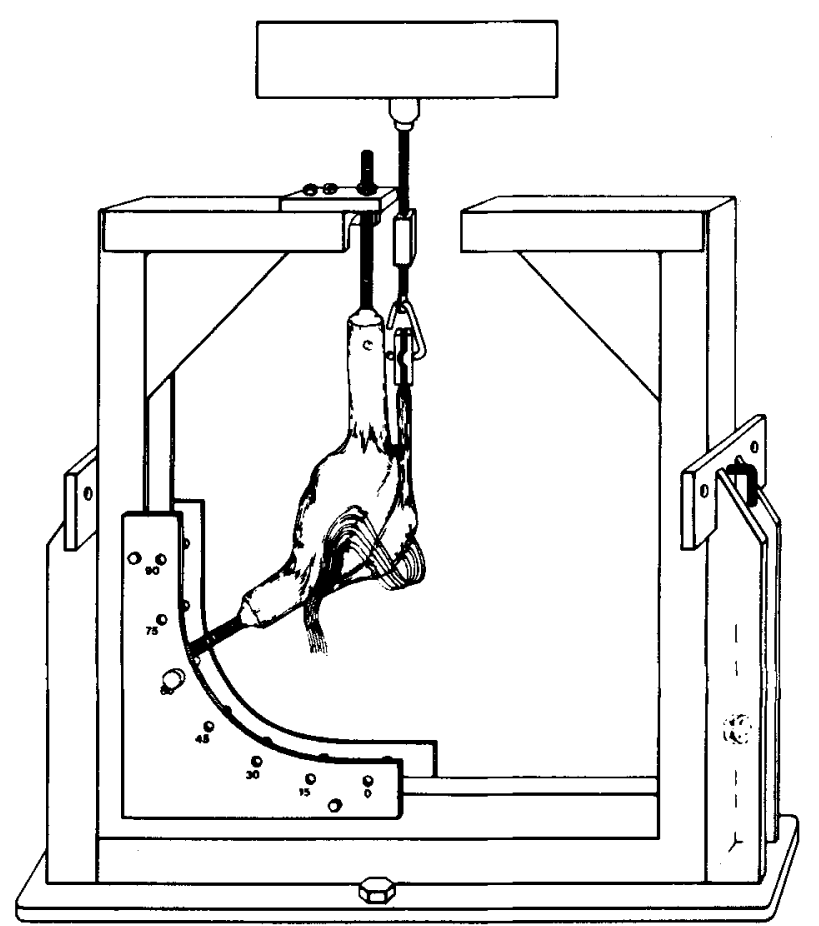

FIG. 2. The experimental loading apparatus was located on the bed of an Instron Universal testing machine. 
Universal testing machine provided at least $1,000 \mathrm{~N}$ of simulated quadriceps force without slippage. This device was stationed on an axis that allowed the quadriceps load direction to be altered by $12^{\circ}$ to the medial or lateral side of the natural position.

Approximately physiological $\mathrm{Q}$ angles were determined by aligning the quadriceps tendon and the loading clamp with the femoral shaft. Flexion of the knee could be adjusted from full extension to $90^{\circ}$ of flexion by $15^{\circ}$ increments. Quadriceps load was monitored by a strain gage load cell within the Instron loading actuator. Although quadriceps load and patellar tendon load may not be equal at all knee flexion angles (12), we chose to monitor patellar strain as a function of quadriceps load as an experimentally reproducible and useful method that best relates to available reports of patellofemoral joint function. All strain and load data were acquired directly on a Tektronix 4054 (Beaverton, OR, U.S.A.) computer system via an analog to digital converter. Prior to each test the strain gages and load cell were calibrated using known shunt resistors built into the instrumentation system.

Two main experiments were performed. The first experiment used five matched pairs of knees tested intact with normal patellofemoral articulations and retested after total knee arthroplasty with patella resurfacing. One knee from each pair was reconstructed using a resurfacing total knee arthroplasty with domed patellar component (Total Condylar, Howmedica Inc., Rutherford, NJ, U.S.A.). The contralateral knee was reconstructed using a linked arthroplasty (Spherocentric, Howmedica Inc.) with a bifaceted congruent patellar component with two fixation posts (Fig. 3). Throughout the range of motion, the domed patella demonstrated a smaller area of contact with the patellar flange when compared with the bifaceted component. All knees were tested at quadriceps loads from 0 to $1,000 \mathrm{~N}$ and at flexion angles of 0 to $90^{\circ}$ at $15^{\circ}$ increments. The pa- tellofemoral alignment remained neutral during the experimental procedures.

The second experiment was performed using seven pairs of cadaver knee joints. All knees were tested with the natural patella at quadriceps loads of 0 to $1,000 \mathrm{~N}$, at three quadriceps angles (neutral, $12^{\circ}$ lateral, $12^{\circ}$ medial) and in flexion from 0 to $90^{\circ}$ in $15^{\circ}$ increments. After testing, total knee arthroplasty was performed on all knees using a standard resurfacing implant (Total Condylar) and no patellar component. The complete series of tests was then repeated.

After these tests, patellar components were implanted. One knee from each pair received the standard polyethylene button component; the other received a custom-made button with stainless steel metal backing. The tests were then repeated.

In both experiments each knee served as its own control. All arthroplasties were performed using standard surgical technique and methylmethacrylate fixation. A medial parapatellar approach was used and the capsule was closed anatomically after the surgery. Care was taken to ensure that the patella tracked smoothly through a complete range of motion.

The experimental design provided 1,872 records of patellar strain as a function of location on the patella, quadriceps load, knee flexion angle, quadriceps angle, total joint arthroplasty with and without patellar replacement, patellar prosthesis design including fixation peg location, patellofemoral congruity, and metal backing.

\section{RESULTS}

There was a linear relationship between the quadriceps load and the measured strain on the anterior surface of the patella at all flexion angles. Although several knees were loaded until grip failure

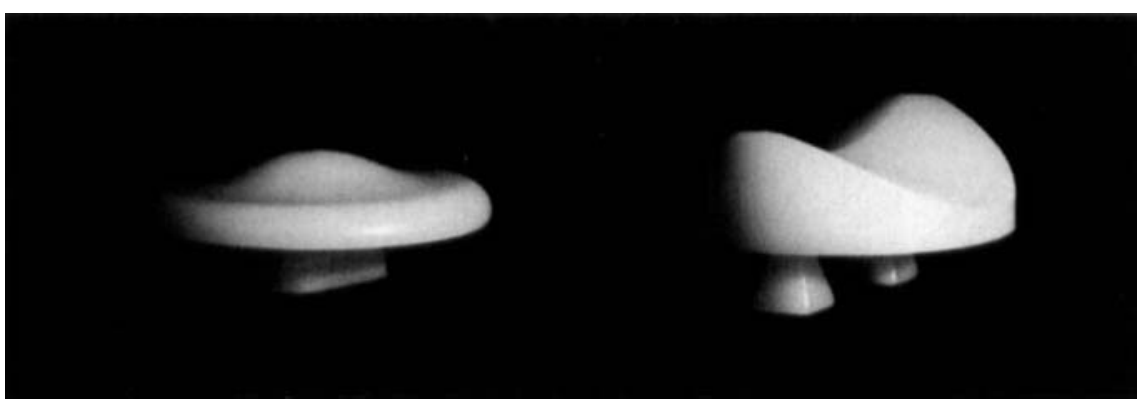

FIG. 3. The two patellar components used in this study are illustrated above. The domed single fixation peg component demonstrates a smaller area of contact when compared with the bifaceted patella with two fixation pegs. 
$(\sim 2,000 \mathrm{~N})$, this relationship still remained linear (Fig. 4).

In general, the greatest strains were recorded at the distal gage location (Fig. 5). The variance of the data, however, was very large when all knees were grouped together as illustrated in Fig. 6. Although the individual specimens demonstrated similar patterns of strain, these were masked by the standard deviations associated with the pooled data. To better interpret these strain patterns, a simple normalization procedure was applied to the data. For each knee, at a given load level, the maximal strain of each gage was assigned the value of $100 \%$. All strain values subsequently were calculated relative to this value. After the total joint procedures, the strain was also evaluated relative to the maximal value for the intact knee. Therefore, increases in microstrain caused by the total joint components could be represented by values $>100 \%$. All subsequent results are expressed in this normalized format.

Mean maximal strains occurred in the intact knees at flexion angles between 45 and $60^{\circ}$ with greater distal strain experienced at lower flexion angles and greater proximal strain at greater flexion angles. All measured strains were least near full extension. This finding was consistent for all three gage locations. A lateral and medial deviation in quadriceps angle did not cause significant reductions in the microstrain values at any of the flexion angles (Fig. 7).

Polyethylene prosthetic surface replacement of the patella caused significant increases in surface strain at the proximal and middle positions. Specifically, the button prosthesis produced strain that may approach fracture levels, especially when con-

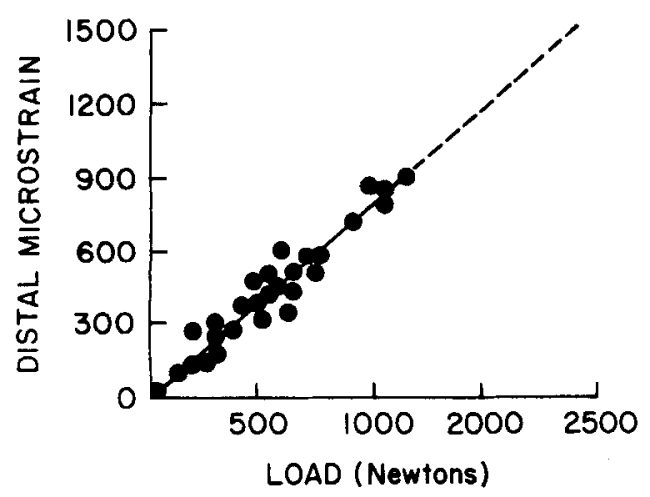

FIG. 4. Four knees were loaded until grip failure. Each exhibited a linear relationship between strain and quadriceps load illustrated by the response of one knee.

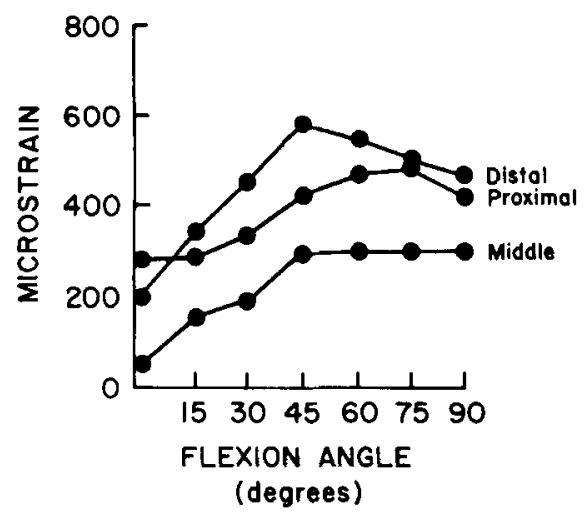

FIG. 5. The mean strain for all knees is illustrated as a function of flexion angle.

sidering fatigue. The bifaceted component, however, demonstrates more nearly normal strain levels (Fig. 8).

When total joint arthroplasty was performed with no patellar resurfacing, there was little increase in the recorded microstrain at all gage locations (Fig. 9). The results indicate that whereas patellar button resurfacing significantly increases microstrain at the proximal and middle locations, a metal backing can reduce this effect (Fig. 9). This metal backing effect, however, was not found at the distal (Fig. 10) gage location.

\section{DISCUSSION}

Although limited to three locations, we believe that our measurements of anterior patellar surface strain may provide experimentally determined indices or check points for more comprehensive analytic models of the patella or the patellofemoral joint. These analytic models may extend our pre-

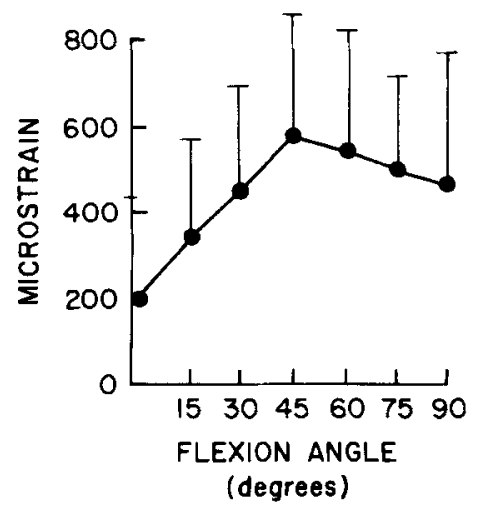

FIG. 6. The biologic variability between specimens is demonstrated by the standard deviations associated with the pooled data. This is demonstrated by the averaged data for the distal strain gage location. 


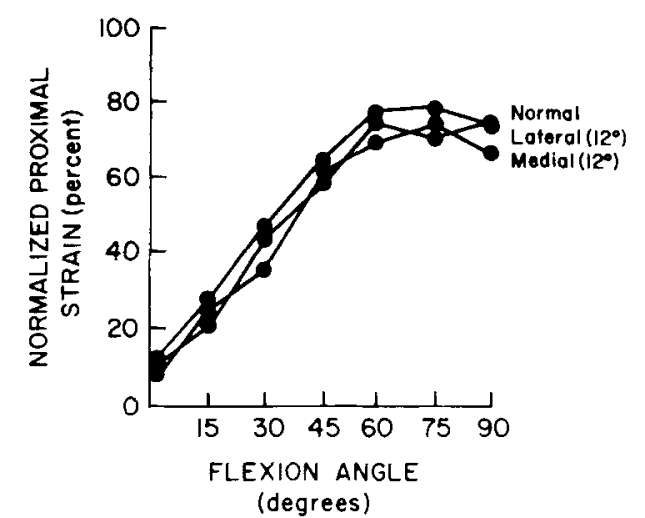

FIG. 7. The effect of quadriceps angle is presented above for the promixal gage location. The change in strain was not statistically significantly different at any of the gage locations.

dictive understanding not only to measurable surface locations but also to trabecular and subchondral bone. At the present time, however, the relationship between the surface strains and local stresses in the subchondral bone is not known and would require further study. These studies can, in addition, predict the response of the patella to a variety of experimental, pathologic, or therapeutic conditions. Our studies may also provide quantitative guidelines for the development of patellar or patellofemoral prostheses so as to minimize the mechanical alterations to the supporting bone.

Using functional quadriceps loads previously reported $(21,25)$, the functional maximal strain during normal activity calculated from our data is between 1,000 and 2,000 microstrain. This correlates well with reported physiologic values of cortical bone surface strain $(2,16,22,23)$.

The results of our experiments are presented as a

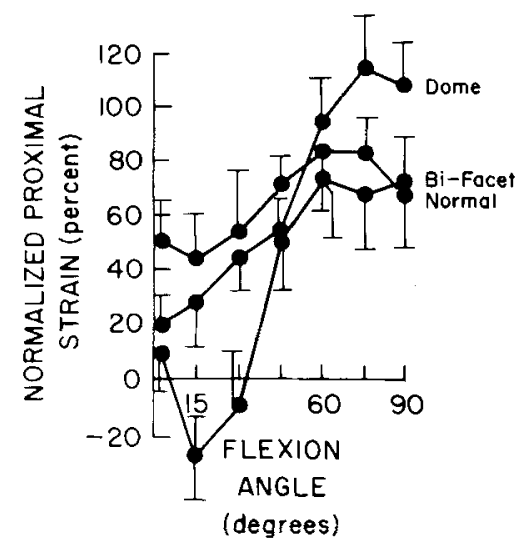

FIG. 8. Resurfacing the patella with a domed and bifaceted polyethylene component increased strain in the proximal and middle locations. The results from the proximal gage are illustrated.

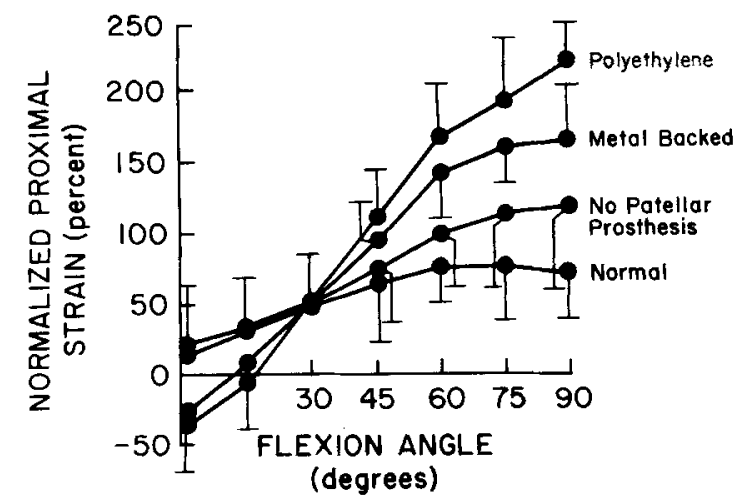

FIG. 9. The effects of a metal backed component are illustrated at all gage locations. Total knee arthroplasty with and without patellar resurfacing on proximal strain is demonstrated.

function of quadriceps load. From the work of $\mathrm{Hu}$ berti et al. (12), it is important to note that the force ratio between the quadriceps mechanism and ligamentum patellae was found to be a function of knee flexion angle. Below $45^{\circ}$, the force in the ligamentum patellae was greater than in the quadriceps tendon. At flexion angles greater than $45^{\circ}$ this phenomena reversed. Our surface strain data tended to follow this trend. The average distal strain was greater than the proximal strain until it peaked at $45^{\circ}$. Between 45 and $90^{\circ}$ the distal strain decreased significantly. The proximal strain also peaked between 45 and $60^{\circ}$ but only decreased slightly after $60^{\circ}$.

In general, the results of this study suggest that alterations in quadriceps force angle have little effect on surface strain. These findings, however, would need to be verified by similar experiments with strain gages at sites other than on the midline of the patella.

A conforming patellar prosthesis supported over its entire functioning surface and having peripheral

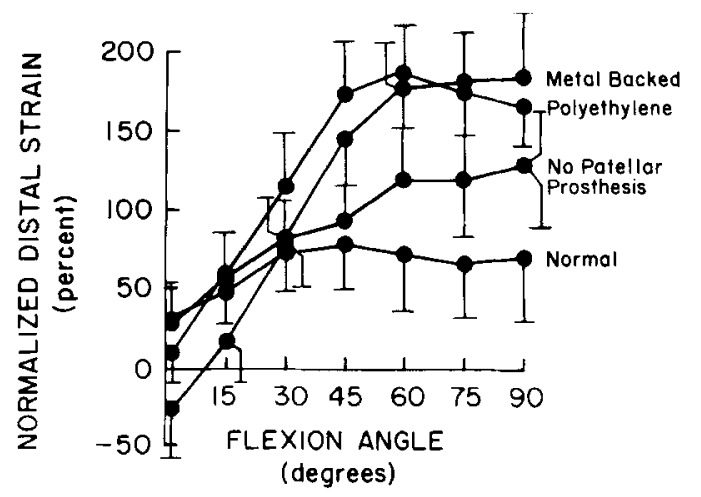

FIG. 10. At the distal gage location, the effect of metal backing was insignificant. 
fixation pegs increases the patellar surface strain far less than a patellar button with a central peg. These effects may be attributed to both the difference in patellofemoral flange congruity and the central versus dual fixation peg designs (Figs. 8 and 9). The relative contribution of these two design parameters, however, cannot be evaluated from this study.

Under most circumstances, the natural patella functioning against a prosthetic patellofemoral flange demonstrates far less surface strain than patellas treated with prostheses. This would seem to indicate that the interaction of the normal patella and a femoral component flange would subject the patella to the least damaging mechanical alterations. Clinical indices of pain, degeneration, and function, however, may necessitate resurfacing. Metal backing of the patellar implant appeared to protect the residual bony patella. The protection was not apparent at the distal gage location. An explanation for this phenomena may be related to the geometry of the implant. The distal gage location was directly beneath the bottom edge of the metal backing, which may have created a stress-concentrating edge effect.

\section{CONCLUSIONS}

These studies of midvertical anterior patellar surface strains indicate that maximum strains occur at angles between 45 and $60^{\circ}$ of knee flexion. Changes in quadriceps angle decrease midvertical strains significantly. All tested polyethylene replacement patellar prostheses increased recorded strains, but a bifaceted, more congruent design caused less increase of strain. Metal support for the polyethylene patellar prostheses protected the residual patella and decreased surface strains at the upper and middle gage locations.

We believe that information from this study and future studies using strain measures from other patellar surface locations should be useful not only for interpreting the effect surgical alterations have in the patellar mechanisms but also in the design of more satisfactory patellar prosthesis.

Acknowledgment: This study was supported in part from a grant from the Orthopaedic Research and Education Foundation.

\section{REFERENCES}

1. Cameron HU, Fedorkow DM: The patella in total knee arthroplasty. Clin Orthop 165:197-199, 1982

2. Carter DR, Schwab GH, and Spengler DM: Tensile fracture of cancellous bone. Acta Orthop Scand 51:733-741, 1980
3. Ferguson AB, Brown TD, Fu FH, Rutkowski R: Relief of patello-femoral contact stress by anterior displacement of the tibial tubercle. J Bone Joint Surg [Am] 61:159-166, 1979

4. Ficat RP, Hungerford DS: Disorders of the Patello-Femoral Joint. Baltimore, Williams and Wilkins, 1977

5. Goldstein SA, Weiss A-PC, Coale E, Matthews LS: Surface strain studies of the human patella. In: Transactions of the 30th Annual Orthopaedic Research Society, Atlanta, GA, 9:26, Feb, 1984

6. Goodfellow J, Hungerford DS, Zindel M: Patello-femoral joint mechanics and pathology. J Bone Joint Surg. $[\mathrm{Br}]$ $58: 287-290,1976$

7. Hayes WC, Levine BM: Finite element analysis of patellar resurfacing procedures. In: Transactions of the Orthopaedic Research Society, 5:163, 1980

8. Hayes WC, Synder B, Levine BM: Stress-morphology relationships in trabecular bone of the patella. In: Finite Elements in Biomechanics, ed by BR Simon, New York, Wiley, $1980,107-127$

9. Hensal F, Nelson T, Paulou H, Tong JS: Bilateral patellar fractures from indirect trauma. A case report. Clin Orthop 178:207-209, 1983

10. Higgins TT: Fracture of both patellae by muscular action. Br Med J 1:1006, 1925

11. Huberti HH, Hayes WC: Patello-femoral contact pressures. J Bone Joint Surg [Am] 66:715-724, 1984

12. Huberti HE, Hayes WC, Stone JL, Shybut JT: Force rates in the quadriceps tendon and ligamentum patellae. $J$ Orthop Res 2:49-54, 1984

13. Hungerford DS, Barry M: Biomechanics of the patello-femoral joint. Clin Orthop 144:9-15, 1979

14. Kaufer H: Mechanical function of the patella. $J$ Bone Joint Surg $[A m]$ 53:1551-1556, 1971

15. Kettlekamp DB: Management of patellar malalignment current concepts review. J Bone Joint Surg [Am] 63:13441348,1981

16. Lanyon LE, Goodship AE, Baggot DG: Significance of bone strain in vivo. Acta Orthop Belg 42, 1976

17. McCarroll IR: Fracture of the patella during a golf swing following reconstruction of the anterior cruciate ligament. A case report. Am J Sports Med 11:26-27, 1983

18. Maquet P: Un Traitment Biomecanique de L'arthrose Femoro-Patellaire. L'avancement du Tendon Rotulien. Rev Rhum Mal Osteoartic 30:779-783, 1963

19. Matthews LS, Sonstegard DA, Henke JA: Load bearing characteristics of the patello-femoral joint. Acta Orthop Scand 48:511-516, 1977

20. Minns RJ, Birnie AJM, and Abernathy PJ: A stress analysis of the patella and how it relates to patellar articular lesions. J Biomech 12:699-711, 1979

21. Morrison JB: The mechanics of the knee joint in relation to normal walking. $J$ Biomech 3:51-61, 1970

22. O'Connor JA, Lanyon LE: The effect of strain rate on mechanically adaptive bone remodeling. In: Transactions of the Orthopaedic Research Society, New Orleans, 1982, p 103

23. Reilly DT, Burstein AH: The mechanical properties of cortical bone. J Bone Joint Surg [Am] 56:1001-1022, 1974

24. Reilly DT, Martens M: Experimental analysis of the quadriceps muscle force and patello-femoral joint reaction force for various activities. Acta Orthop Scand 43:127-137, 1972

25. Smidt GI: Biomechanical analysis of knee flexion and extension. J Biomech 6:87-92, 1973

26. Steinke C: Simultaneous fracture of both patellae. Ann Surg $58: 510-525,1913$

27. Thompson RM, Hood RW, Insall J; Patellar fractures in total knee arthroplasty. Orthop Trans 5;490, 1981

28. Townsend PR, Rose RM, Radin EL, Raux P: The biomechanics of the human patella and its implications for chondromalacia. J Biomech 12:699-711, 1979 